\title{
Energy Transfer in Master Equation Simulations: A New Approach
}

\author{
JOHN R. BARKER
}

Department of Atmospheric, Oceanic, and Space Sciences, University of Michigan, Ann Arbor, MI 48109-2143

Received 5 February 2009; revised 14 April 2009, 19 June 2009; accepted 22 June 2009

DOI 10.1002/kin.20447

Published online in Wiley InterScience (www.interscience.wiley.com).

\begin{abstract}
Collisional energy transfer plays a key role in recombination, unimolecular, and chemical activation reactions. For master equation simulations of such reaction systems, it is conventionally assumed that the rate constant for inelastic energy transfer collisions is independent of the excitation energy. However, numerical instabilities and nonphysical results are encountered when normalizing the collision step-size distribution in the sparse density of states regime at low energies. It is argued here that the conventional assumption is not correct, and it is shown that the numerical problems and nonphysical results are eliminated by making a plausible assumption about the energy dependence of the rate coefficient for inelastic collisions. The new assumption produces a model that is more physically realistic for any reasonable choice of collision step-size distribution, but more work remains to be done. The resulting numerical algorithm is stable and noniterative. Testing shows that overall accuracy in master equation simulations is better with this new approach than with the conventional one. This new approach is appropriate for all energy-grained master equation formulations. (c) 2009 Wiley Periodicals, Inc. Int J Chem Kinet 41: 748-763, 2009
\end{abstract}

\section{INTRODUCTION}

Collisional energy transfer is a key feature of most chemical reaction systems. Collisions activate and deactivate molecular species while establishing the Boltzmann energy distribution. At low pressures, collisional energy transfer is slow and therefore controls the rate of unimolecular reactions. The collision-reaction master equation has proved to be very effective in describing unimolecular reactions in their many variations [1-3], which are categorized according to how the reaction is initiated: thermal activation, photoactivation,

\footnotetext{
Correspondence to: John R. Barker; e-mail: jrbarker@umich. edu.

Contract grant sponsor: NASA (Planetary Atmospheres).

Contract grant sponsor: NSF (Atmospheric Chemistry).

(c) 2009 Wiley Periodicals, Inc.
}

chemical activation, etc. In all of these, collisional energy transfer plays an important role.

As discussed elsewhere [4,5], energy transfer involving polyatomic molecules was first investigated as a part of unimolecular reaction studies, but was later investigated in nonreactive systems by "direct" timeresolved experimental techniques based on, for example, spontaneous infrared fluorescence [6-8], ultraviolet [9] or infrared [10] absorption, and photoionization [11]. In recent advances, large molecule energy transfer is being investigated using molecular beam scattering experiments [12]. From all of these experiments, information has been gathered about the average amount of energy transferred per collision, including its magnitude, energy dependence ( $\sim$ linear) and temperature dependence ( $\sim$ constant or perhaps $\sim$ linear) [5]. Other experiments have shown that individual vibrational modes are activated and deactivated more rapidly than 
others $[13,14]$. In many systems, the exponential-down model for the collision step-size distribution (which is discussed below) provides an adequate description, but more detailed experiments have shown that a doubleexponential or a "stretched" exponential model gives a better description [15-17]. In almost all of the studies cited in this paragraph, the researchers analyzed their data on the basis of models that assumed that the inelastic collision frequency is independent of the excitation energy resident in the colliding molecules.

To be clear, unimolecular reactions and master equations can be formulated in terms of the "active energy," which, for symmetric tops, consists of the K-rotor and the vibrations (and internal rotations, if present); because of angular momentum conservation in the unimolecular reactions, the two-dimensional (2D) rotation (of a symmetric top) is omitted from the density of states calculation in the most common treatments of angular momentum [1,3]. Since the same density of states is used in the detailed balance expression for inelastic collisions, the energy transfer parameters refer to the active energy and not to pure vibrational or rotational transitions. In most cases, the collision frequency for changing the active energy has been estimated by assuming Lennard-Jones forces between the collision partners, but it is well known that the total collision frequency is much larger $[18,19]$. In fact, even the collision frequency for pure rotationally inelastic collisions is much larger [20]. It also has long been known that the rate constant for inelastic energy transfer depends on the energy difference between initial and final states, as well as on varying propensities for collisions $[14,20-22]$. Since the average density of states, which depends on internal energy, is closely related to the average energy separation between adjacent states, it is not surprising that experiments show that the total inelastic collision frequency depends on excitation energy [23]. Evidence for collision "propensities" suggests mode-specific behavior [14]. However, this sort of detailed energy transfer information is available for very few molecular species and is therefore not yet of general utility. Fortunately, single-channel unimolecular reaction rates depend mostly on the average rate of energy transfer and are relatively insensitive to the exact details of the models for collision frequency and collision step-size distribution [1-3].

Master equation models can, or course, be tailored to individual molecules when the requisite energy transfer data are available. However, to the best of our knowledge, the complete set of necessary data is not available for any polyatomic species. In the interest of pragmatism, master equation codes intended for general use must be flexible enough so that they can be applied to most polyatomic species and perform with reasonable quantitative accuracy. It is necessary that they can incorporate the main properties of energy transfer: the dependence on temperature and internal energy and the shape of the collision step-size distribution function. Modern codes such as MultiWell [24,25] succeed in this regard by allowing a wide range of optional choices of collision step-size distribution function and parameterization, including the double exponential and the "stretched" exponential mentioned above. A second fundamental requirement is that they accurately simulate the Boltzmann thermal energy distribution, which is needed to predict accurate reaction rate constants. As shown below, current methods succeed in this regard only at relatively high internal energies: they perform poorly at the low energies that are important for low threshold chemical reactions (e.g., the isomerization of trans-stilbene [26] and the dissociation of prereactive complexes) and for shock tube simulations.

The purpose of the present paper is to describe a new approach to the treatment of inelastic collisions that abandons the physically incorrect assumption that the inelastic collision frequency is independent of internal energy and replaces it with an assumption that is qualitatively accurate, although still arbitrary. The resulting simulations of the Boltzmann distribution are significantly more accurate, and the representation of energy transfer at low energies is significantly more realistic than before. At higher energies, the results are quantitatively very similar to the traditional treatment. Because the new assumption is arbitrary (although qualitatively reasonable), this approach is only an interim solution. But breaking with tradition on the inelastic collision frequency produces a more accurate physical model as well as a more accurate master equation code.

\section{THEORY}

\section{The Master Equation}

The collision-reaction master equation [1-3] can be written using Forst's simplified notation [1], where precollision and postcollision energies are written $y$ and $x$, respectively:

$$
\begin{aligned}
\frac{\mathrm{d} W(y, t)}{\mathrm{d} t}= & F(y, t)+\int_{0}^{\infty} R(y, x) W(x, t) \mathrm{d} x \\
& -\int_{0}^{\infty} R(x, y) W(y, t) \mathrm{d} x \\
& -\sum_{i=1}^{\text {channels }} k_{i}(y) W(y, t)
\end{aligned}
$$


In Eq. (1), $W(y, t) \mathrm{d} y$ is the concentration of a chemical species with vibrational energy in the range $y$ to $y+\mathrm{d} y ; R(x, y)$ is the (pseudo-first-order) rate coefficient for collisional energy transfer from initial energy $y$ to energy $x ; F(y, t) \mathrm{d} y$ is a source term (e.g., thermal, chemical, or photo activation); and $k_{i}(y)$ is a unimolecular reaction rate constant for molecules at energy $y$ reacting via the $i$ th reaction channel. Other terms, such as those involving radiative emission and absorption have been omitted.

The rate coefficient $R(x, y)$ is conventionally written as the product of the total vibrationally inelastic collision frequency $k_{c}(y)[M]$ multiplied by the "collision step-size distribution," $P(x, y)$, which expresses the probability density that a molecule initially with initial energy $y$, will undergo an inelastic transition to the energy range $x$ to $x+\mathrm{d} x$ :

$$
\begin{aligned}
R(x, y) \mathrm{d} x & =\int_{0}^{\infty} R(x, y) \mathrm{d} x\left\{\frac{R(x, y) \mathrm{d} x}{\int_{0}^{\infty} R(x, y) \mathrm{d} x}\right\} \\
& =k_{c}(y)[M] P(x, y) \mathrm{d} x
\end{aligned}
$$

The first factor on the right-hand side of Eq. (2a), the integral over the rates of all inelastic transitions from initial energy $y$, is the frequency of inelastic collisions, $k_{c}(y)[M]$, and the second factor (in curly brackets) is $P(x, y) \mathrm{d} x$. Note that $P(x, y)$ is normalized:

$$
\int_{0}^{\infty} P(x, y) \mathrm{d} x=1
$$

It is important to emphasize that the factorization of $R(x, y)$ in Eq. (2) is merely for convenience and that $k_{c}(y)[M]$ and $P(x, y)$ never occur independently of one another. Furthermore, $P(x, y)$ only has an unambiguous physical interpretation when $k_{c}(y)[M]$ is exactly equal to the total inelastic collision rate constant. Although neither the exact inelastic collision frequency nor $P(x, y)$ is known, experimental unimolecular reaction rates and collisional energy transfer data can be fitted by adopting assumed forms for $P(x, y)$ and $k_{c}(y)$. According to convention, $k_{c}(y)$ is assumed to be a constant. The inevitable errors in these quantities tend to compensate for one another when fitting experimental data and thus it is important to use $k_{c}(y)$ and $P(x, y)$ in a matched pair whenever possible [2,3,27].

\section{Detailed Balance}

By considering detailed balance at equilibrium in the absence of reactions, the relationship between $R(x, y)$ and $R(y, x)$ can be found. Detailed balance requires that in every increment of energy, the rates of forward and reverse processes must balance. Therefore,

$$
\begin{gathered}
W(y) R(x, y) \mathrm{d} x=W(x) R(y, x) \mathrm{d} y \\
W(y) k_{c}(y) P(x, y)=W(x) k_{c}(x) P(y, x)
\end{gathered}
$$

where Eq. (4b) has been written by using Eq. (2b) and noting that $\mathrm{d} x=\mathrm{d} y$. At equilibrium in the absence of reaction, the ratio of concentrations is also given by the ratio of Boltzmann factors:

$$
\frac{W(x)}{W(y)}=\frac{\rho(x)}{\rho(y)} \exp \left[-(x-y) / k_{B} T\right]
$$

where $\rho(x)$ is the density of states at energy $x$. By using Eqs. (4b) and (5), we obtain the detailed balance relationship between the probability densities for upand down-collisions:

$$
\frac{P(x, y)}{P(y, x)}=\frac{k_{c}(x)}{k_{c}(y)} \frac{\rho(x)}{\rho(y)} \exp \left[-(x-y) / k_{B} T\right]
$$

\section{Collision Step-Size Distributions}

The total probability density for an energy changing collision is normalized (see Eq. (3)) and can be written as the sum of two integrals corresponding to down- and up-collisions:

$$
1=\int_{0}^{y} P(x, y) \mathrm{d} x+\int_{y}^{\infty} P(x, y) \mathrm{d} x
$$

To construct a normalized collision step-size distribution (the probability density), it is common practice to specify a (dimensionless) nonnormalized function $f(x, y)$, which is assumed to be proportional to $P(x, y)$ :

$$
P(x, y)=\frac{f(x, y)}{N(y)}
$$

where $N(y)$ is a normalization constant so that $P(x, y)$ satisfies Eqs. (3) and (7). With this definition, the normalization Eq. (7) becomes

$$
1=\int_{0}^{y} \frac{f(x, y)}{N(y)} \mathrm{d} x+\int_{y}^{\infty} \frac{f(x, y)}{N(y)} \mathrm{d} x
$$

After rearranging Eq. (9), we obtain a formal expression for the normalization constant $N(y)$, expressed as a sum of integrals:

$$
N(y)=\int_{0}^{y} f(x, y) \mathrm{d} x+\int_{y}^{\infty} f(x, y) \mathrm{d} x
$$


or

$$
N(y)=N_{d}(y)+N_{u}(y)
$$

where subscripts $d$ and $u$ denote down-steps $(x<y)$ and up-steps $(x>y)$, respectively.

After utilizing Eq. (8), the expression for detailed balance (Eq. (6)) takes the following form:

$$
\frac{f(x, y)}{f(y, x)}=\frac{N(y)}{N(x)} \frac{k_{c}(x)}{k_{c}(y)} \frac{\rho(x)}{\rho(y)} \exp \left[-(x-y) / k_{B} T\right]
$$

For convenience, the unnormalized function $f(x, y)$ is usually specified for down-steps, but one could choose to specify a function for up-steps instead. We will follow convention and specify the function for downsteps, $f(x, y)=f_{d}(x, y)$ with $x<y$. Thus $N_{d}(y)$ is easily evaluated:

$$
N_{d}(y)=\int_{0}^{y} f_{d}(x, y) \mathrm{d} x
$$

and $N_{u}(y)$ can be expressed in terms of $f_{d}(x, y)$ by using Eq. (12):

$$
\begin{aligned}
N_{u}(y)= & \int_{y}^{\infty} f_{d}(y, x) \frac{N(y)}{N(x)} \frac{k_{c}(x)}{k_{c}(y)} \frac{\rho(x)}{\rho(y)} \\
& \times \exp \left[-(x-y) / k_{B} T\right] \mathrm{d} x
\end{aligned}
$$

If we had assumed that $f(x, y)$ was specified for upsteps, an analogous procedure would be followed.

Since $N(x)$ appears in the integral expression for $N_{u}(y)$, the solution of Eq. (14) is not completely straightforward. Normalization constant $N(y)$ can be found by using trial values for $N(x)$ and employing an iterative solution [28] of Eq. (14), or by rearranging the equation as follows: inelastic collision rate constants are independent of internal energy: $k_{c}(y)=k_{c}(x)=$ constant $[1,3,28,29]$. This constant is conventionally identified with $k_{\mathrm{LJ}}$, the bimolecular rate constant for collisions between particles governed by a Lennard-Jones intermolecular potential [1-3]. Experience has shown that $k_{\mathrm{LJ}}$ is a reasonable approximation to the rate constant for active-energy-changing inelastic collisions involving large molecules (i.e., with high density of states) with internal energies much greater than $k_{B} T$ [30-33]. With this assumption, the ratio of collision rate constants in Eqs. (12), (14), and (15) is equal to unity. (This conventional assumption was used in all versions of MultiWell prior to v.2009.0.)

\section{Problems with Normalization}

Experience has shown that iterative normalization [28] converges reasonably rapidly at high energies, but problems emerge at low energies, where the density of states is sparse and has large relative fluctuations. The problems are most severe when an energy grain that contains just a few states is bracketed on both sides by energy grains containing much higher densities of states. Several examples of this behavior are seen in Fig. 1. For these cases, the normalization factors for some of the energy grains tend to diverge, instead of converging during the iterative calculation. Because of this problem, it has been necessary to limit the number of iterations to, e.g., 2-5, so that normalization at high energy converges sufficiently, while normalization at low energy does not diverge too much. In previous work, more iterations were sometimes used to achieve better performance in certain energy ranges [34]. As the number of iterations is increased, results at higher energies systematically become more accurate, while those at low energies become less so. If the

$$
N(y)=\frac{\int_{0}^{y} f_{d}(x, y) \mathrm{d} x}{1-\int_{y}^{\infty} \frac{f_{d}(y, x)}{N(x)} \frac{k_{c}(x)}{k_{c}(y)} \frac{\rho(x)}{\rho(y)} \exp \left[-(x-y) / k_{B} T\right] \mathrm{d} x}
$$

Equation (15) can be solved with the finite difference algorithm described by Gilbert and coworkers [2,29]. Both of these approaches to finding $N(y)$ are based on specifying $f_{d}(x, y)$ and requiring that $N(y)$ first be estimated at very high energies, well above the energies of interest, where $N_{d}(y)$ and $N_{u}(y)$ tend to become independent of energy (at least when the average energy transferred per collision is independent of energy).

In general, $k_{c}(y)$ is expected to depend on the initial energy, $y$, but it is common practice to assume that the number of iterations is not constrained, the normalization factors calculated for some individual energy grains at low energies appear to approach $\infty$ and can experience numerical overflows. The strategy of limiting the number of iterations, although not completely satisfactory, is reasonably effective in producing steady-state energy distributions that simulate the equilibrium Boltzmann distribution; and example of the results obtained in this way is shown later in Fig. 3. 


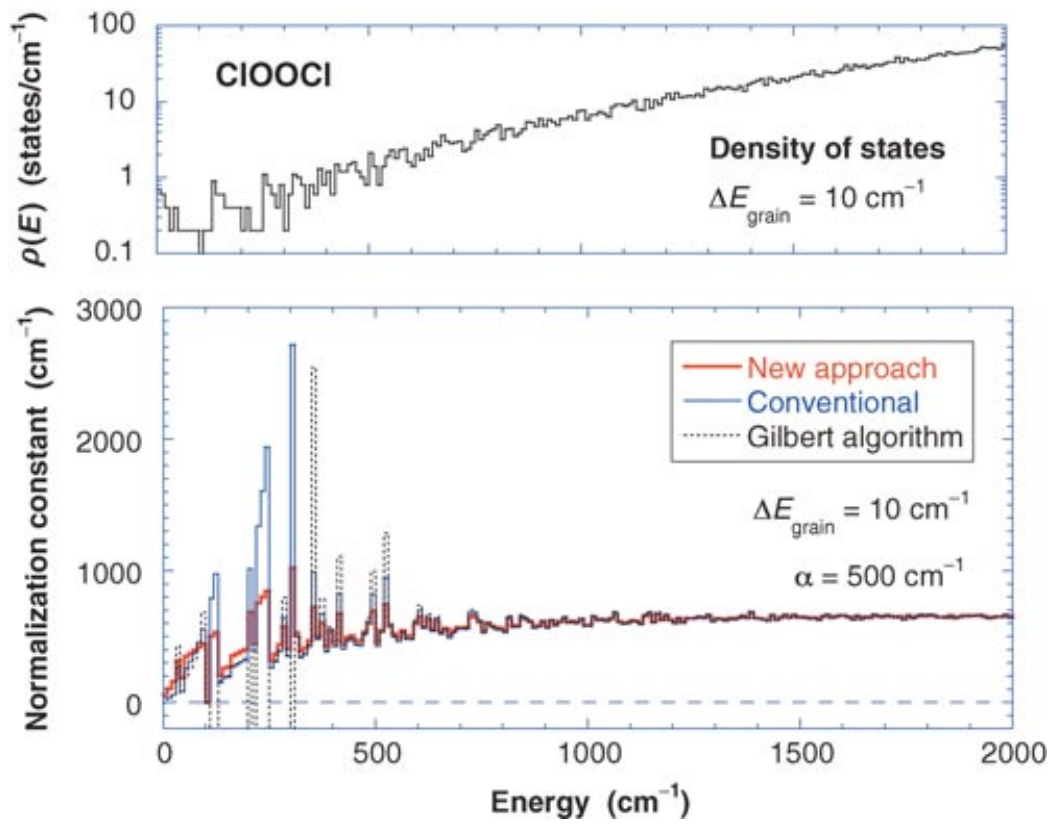

Figure 1 Normalization constants (lower panel) computed using the method of Gilbert and coworkers [2,29], the conventional (iterative) approach [40], and the new approach. Densities of states are shown in the upper panel. Note that the energy grain at $100 \mathrm{~cm}^{-1}$ contains no states and one of the negative excursions (clipped at the lower plot boundary) extends to almost $-3000 \mathrm{~cm}^{-1}$. At higher energies the three normalization approaches become indistinguishable. [Color figure can be viewed in the online issue, which is available at www.interscience.wiley.com.]

Related problems arise at low energies when using Eq. (15) and the finite difference algorithm of Gilbert and coworkers $[2,29]$. The Gilbert algorithm is quite general, but in practice the pragmatic assumption is made that the collision frequency is independent of excitation energy. The algorithm is very accurate at higher energies, but it is not convenient for use with a continuous master equation such as MultiWell, which interpolates between widely separated energies at high excitation energies and adopts a contiguous set of energy grains at low energies. In addition, the Gilbert algorithm does not assign a value for $N(y=0)$. Aside from these considerations, the Gilbert algorithm does not perform well at low energies for the standard exponential-down model when assuming that $k_{c}(y)$ is independent of energy (i.e., the conventional approach). An example of this poor behavior is shown in Fig. 1, where the excursions of the normalization constant are not only large (clipped at the boundary of the plot), but negative and therefore unphysical. It is also significant that the negative excursions occur at the same energies as the large positive excursions obtained using the iterative method described above.

For most master equation simulations, the lowest relevant energies are a few $k_{B} T$ below the reaction threshold energy, which is high enough so that the normalization problems do not emerge. However, in shock tube simulations [34-36] and in simulating reactions with very low energy barriers [26] the relevant energies extend all of the way to the bottom of the energy ladder. Furthermore, in 2D $(E, J)$ master equation simulations, the normalization problems are exacerbated because the $2 \mathrm{D}$ density of states is even more sparse and hence exhibits even greater fluctuations than in the one-dimensional (1D) version.

One solution to these problems is to forego the use of direct counts of states and to instead use smooth approximations such as the Whitten-Rabinovitch approximation [37] or the method of steepest descents [1]. Forst has pointed out that smooth approximations are more appropriate for computing microcanonical rate constants, $k(E)$, from RRKM theory, because the theory is based on semiclassical approximations [1]. However, a fine-grained density of states function is needed to achieve accurate agreement with the quantum Boltzmann distribution when nonreactive energy transfer is of key importance, as in shock tube simulations and in the reactions of weakly bound complexes. For that reason, we prefer to use methods based on exact counts of states whenever possible.

One possible explanation of the large positive (or negative) excursions is sensitivity to numerical noise. This possibility is apparent in Eq. (15). At $y=0$, the zero-point energy, both the numerator and the denominator in Eq. (15) are identically equal to zero. At low energies above zero, the denominator is small 
and the expression is sensitive to numerical errors. As the energy grain size is made smaller, the sensitivity becomes larger. However, numerical noise is minimal when using a double-precision version of the Gilbert algorithm, and it is not the only cause of the large negative excursions.

Because the Gilbert algorithm is based on very general principles [2,29] and still produces unphysical results suggests that there is a fundamental deficiency in the conventional model for collisional energy transfer. The only feature of the conventional model that is not required by the Gilbert algorithm is the conventional assumption that the inelastic collision rate constant $k_{c}(y)$ is independent of energy, $y$. Thus we identify this assumption with the unphysical results. In the next section, we argue that the inelastic collision frequency should vary significantly with energy, especially at low energies. A simple revision to the conventional approach is then proposed, which eliminates the numerical problems with normalization, yields an energydependent inelastic collision frequency, and produces more accurate simulations of the Boltzmann distribution at low energies. Although this revision is arbitrary, it is at least physically plausible and behaves in a qualitatively correct manner.

\section{REVISIONS TO THE NORMALIZATION ALGORITHM}

As pointed out above, $k_{c}(y)$ is expected to depend on internal energy, $y$. Both experiments and theories of state-to-state inelastic energy transfer show that the rate of inelastic collisional energy transfer is very slow when adjacent states are widely separated in energy, but the rate becomes very fast when state densities are high $[3,5,13,20]$. Put another way, the rate of inelastic collisions is very small in the state-to-state region at low energy and rather large at high energy where the density of states is high [20]. In standard master equation treatments, elastic collisions are ignored, since they add to the computational burden and do not result in energy changes. Thus the rate constant for inelastic collisions is the relevant quantity for master equation simulations, and it tends to increase as the density of states increases, at least at low energies.

The normalization factor $N(y)$ also tends to increase with energy. This is because the integral over downsteps increases with energy (at least at low energies), whereas the integral over up-steps remains more or less constant or increases with energy as the number of empty energy grains decreases. Typically, $N(y)$ increases from a low value at $y=0$ and becomes constant within a few percent when the density of states reaches $\sim 100$ states $/ \mathrm{cm}^{-1}$ (for $10 \mathrm{~cm}^{-1}$ energy grains and an exponential model with constant energy transfer parameter $\alpha$; see below). This behavior is also seen in a statistical dynamical model energy transfer model published a few years ago [38,39].

We argue that in principle it should be possible to express $k_{c}(y)$ as a function of $N(y)$, but the specific functional dependence is not known. In the absence of specific knowledge about $k_{c}(y)$, we make a simple, pragmatic assumption that $k_{c}(y)$ is directly proportional to $N(y)$. With this assumption, the following ratio, which appears in Eq. (14), equals unity:

$$
\frac{N(y)}{N(x)} \frac{k_{c}(x)}{k_{c}(y)}=1
$$

and Eq. (14) becomes

$$
N_{u}(y)=\int_{y}^{\infty} f_{d}(y, x) \frac{\rho(x)}{\rho(y)} \exp \left[-(x-y) / k_{B} T\right] \mathrm{d} x
$$

This expression, like Eq. (13), requires only straightforward integration. For the purpose of numerical integration, the integral can be converted to a sum and Eq. (17) becomes

$$
\begin{aligned}
N_{u}(j)= & \Delta E_{\text {grain }} \sum_{i=j+1}^{j_{\max }} f_{d}(j, i) \frac{\rho(i)}{\rho(j)} \\
& \times \exp \left[-(i-j) \Delta E_{\text {grain }} / k_{B} T\right]
\end{aligned}
$$

where $i$ and $j$ are index numbers, $j_{\max }$ is the highest energy grain in the model, and $\Delta \mathrm{E}_{\text {grain }}$ is the energy grain size. Because the normalization constant does not exist if there are no states, empty energy grains are omitted when evaluating Eq. (18). Thus, $\rho(j)$ is always $>0$, no singularities are possible, and the normalization constant $N(y)=N_{d}(y)+N_{u}(y)$ is greater than zero and absolutely stable. No iterative methods or finite difference algorithms (i.e., the Gilbert algorithm) are needed for its evaluation. No smoothing of the density of states is required. As a result, the normalization at low energies is never less than zero and it does not show excursions that are as extreme as those found using the conventional (iterative) method, as shown in Fig. 1. In the calculations carried out below, the trapezoidal rule was used instead of the simple sum shown in Eq. (18), but the considerations are the same.

The assumption that $k_{c}(y)$ is directly proportional to $N(y)$ requires that the collision frequency for a molecule with excitation energy $y$ be calculated using $k_{c}(y)=C N(y)$ (see Eq. (16)), where the 
proportionality constant $C$ must be established by some other means. Since the Lennard-Jones rate constant $k_{\mathrm{LJ}}$ conventionally has been used for calculating the low-pressure limit of unimolecular and recombination reactions, we have adopted the following form for the total energy-dependent rate constant for the inelastic collision frequency:

$$
k_{c}(y)=\frac{k_{\mathrm{LJ}}}{N\left(E_{\mathrm{ref}}\right)} N(y)
$$

where $E_{\text {ref }}$ is a reference energy. By combining Eq. (19) with Eqs. (16), (12), (8), and (2) and incorporating the definition of $f_{d}(x, y)$, we obtain the following expressions for the rates of energy transfer:

$$
\begin{aligned}
& \text { Down-steps: } \quad \text { for } x<y \\
& \qquad R_{d}(x, y) \mathrm{d} x=k_{\mathrm{LJ}}[M] \frac{f_{d}(x, y) \mathrm{d} x}{N\left(E_{\text {ref }}\right)} \\
& \text { Up-steps: } \quad \text { for } x<y \\
& \qquad R_{u}(x, y) \mathrm{d} x=k_{\mathrm{LJ}}[M] \frac{\rho(x)}{\rho(y)} \\
& \quad \times \exp \left[-(x-y) / k_{B} T\right] \frac{f_{d}(y, x) \mathrm{d} x}{N\left(E_{\text {ref }}\right)}
\end{aligned}
$$

These expressions are easy to implement since the normalization constant need only be computed at the reference energy $E_{\text {ref }}$. Moreover, since the rates of collisional energy transfer do not depend on $N(y)$, the energy-dependent normalization factor, the population distributions are not affected by errors in normalization.

Although the energy-dependent normalization factor $N(y)$ does not appear in Eq. (20), it is important to examine its behavior. In most cases, $N(y)$ is relatively smooth at energies where the density of states is $\geq 100$ states $/ \mathrm{cm}^{-1}$; this condition provides a good criterion for specifying $E_{\text {ref }}$. Other choices for $E_{\text {ref }}$ will produce numerically different collision frequencies and energy transfer parameters, but above the energy where the density of states becomes relatively smooth, there is little further difference (see Fig. 1), unless the energy transfer parameter $\alpha$ depends on energy. In reaction studies, the rate of energy transfer is most important at energies near the reaction critical energy. For nonreactive systems, the density of states criterion may be used (see Appendix A for the criteria used in the latest version of MultiWell). As discussed below, the numerical results obtained using this new approach are nearly the same as those obtained using the old conventional approach, except at low energies. However, it should be noted that $k_{\mathrm{LJ}}$ may not always be the best choice for $k_{c}$, as discussed in the Introduction. This is especially true for 2D master equation models, in which rotational energy transfer is considered explicitly, since the rate constants for rotationally inelastic collisions are much larger than $k_{\mathrm{LJ}}$.

\section{COMPUTATIONAL METHODS}

It should be emphasized that the new approach is quite generally valid for all energy-grained master equation formulations, whether solved by a stochastic method or by matrix diagonalization. Care must be taken so that energy grains that do not contain any states also never contain any population. Such "empty" energy grains can be omitted from the calculation. For energy grains that do contain states, Eq. (18) provides the basis for a very simple algorithm for computing the normalization factor $N(y)$, since it does not depend on the $N(x)$ for any other energy grain. The Gilbert algorithm is not required: Eq. (18) requires only a simple numerical integration. Of course, the collision rate constant $k_{c}(y)$ varies with energy $y$, as described by Eq. (19).

The present calculations were carried out using versions of the MultiWell Program Suite [24]. The conventional calculations were carried out using version 2008.3, which consists of the free-source code, examples, and MultiWell User Manual that are published as a package on the Internet [40]. Note that version 2008.3 utilizes three (3) iterations when calculating the "conventional" normalization constant, as discussed above. The calculations carried out using the new approach (described in the preceding section) are not iterative and were performed with a development version of MultiWell (which is now available as v.2009 on the MultiWell Web site [25]).

The MultiWell master equation is based on a "hybrid" approach, which consists of a continuum master equation formulation at high energies and an energygrained master equation at low energies. Densities of states and other quantities are stored in "double arrays," where the energy grain at low energy is small enough (typically 5 or $10 \mathrm{~cm}^{-1}$ ) for proper numerical convergence; much larger energy steps (typically $\geq 100 \mathrm{~cm}^{-1}$ ) are stored for higher energies. The dividing line between the upper and lower energy regimes is typically set at the lowest energy where the grain-tograin difference in densities of states is less than a few percent and the density of states function is relatively smooth. The numerical integrations were carried out by using the trapezoidal rule in the lower energy regime. In the upper energy regime, the integrations were carried out analytically for each energy step by noting that the density of states is an approximately exponential 
function of internal energy over the limited range of each energy step.

Once again, it should be emphasized that the new approach to normalization is appropriate for all master equation codes, not just MultiWell.

Densities of states were calculated by exact counts using "Densum," one of the codes included in the MultiWell Program Suite. Densum employs the BeyerSwinehart algorithm [41] as implemented by Stein and Rabinovitch [42] to calculate sums and densities of states based on any combination of separable degrees of freedom consisting of molecular harmonic oscillators, anharmonic oscillators, free internal rotations, hindered internal rotations, and particle-in-a-box. The vibrational frequencies, moments of inertia, and other parameters used for all of the present calculations are summarized in Appendix B.

\section{PERFORMANCE TESTS AND RESULTS}

The standard exponential-down model of energy transfer, which is used in most master equation simulations, is used for the performance tests:

$$
f_{d}(x, y)=\exp \left[\frac{-(y-x)}{\alpha(y)}\right]
$$

where $\alpha(y)$ is a polynomial [5]. In most cases only the constant term of the polynomial is needed to obtain satisfactory fits to unimolecular or recombination reaction rate data, but the linear polynomial coefficient is also needed to accurately fit energy transfer and shock tube data. By adjusting these coefficients, it is possible to compensate, at least in part, for possible errors in the collision frequency (assumed to be given by $k_{\mathrm{LJ}}[M]$ ) and in other elements of the master equation model. It should be emphasized that other energy transfer model functions are more accurate than Eq. (21) for the relatively few cases where the parameters are known [5], as discussed in the Introduction. Many other models (including the double exponential and the stretched exponential model $[15-17,43]$ ) have been investigated by assorted research groups and most of those functions can be selected in MultiWell. In most cases, however, energy transfer parameters are known very poorly, if at all, and thus the Eq. (21) with constant $\alpha$ is the model used by most researchers. Thus it is adopted for the tests presented here.

In this context, it is appropriate to test the accuracy of the present new approach and to determine whether it is still possible to fit experimental data satisfactorily by varying the polynomial coefficients in $\alpha(y)$. Four types of tests have been performed, as described below, with both the old and new approaches.

\section{Boltzmann Distribution}

If the master equation is accurate, simulations for sequences of multiple collisions should converge accurately to the Boltzmann distribution in a thermal system as the number of collisions is increased indefinitely. Expressed in terms of densities of states, the Boltzmann distribution is

$$
B(y ; T)=\frac{1}{Q(T)} \rho(y) \exp \left[-y / k_{B} T\right]
$$

where the partition function $Q(\mathrm{~T})$ is given by

$$
Q(T)=\int_{0}^{\infty} \rho(y) \exp \left[-y / k_{B} T\right] \mathrm{d} y
$$

The average thermal energy is given by

$$
\langle E\rangle_{T}=\int_{0}^{\infty} y B(y ; T) \mathrm{d} y
$$

In each of the first series of tests, master equation simulations were carried out for 1000 random sequences of 1000 collisions each. The initial energy distribution was selected by Monte Carlo techniques from the Boltzmann distribution function. The selection of the initial energy distribution and computation of $\langle y\rangle_{T}$ are standard features in the MultiWell master equation code [24,40], where the integrals in Eqs. (23) and (24) are evaluated numerically with the trapezoidal rule. After every collision, the energy was recorded. This procedure produced $10^{6}$ values per test, which were averaged to obtain the average thermal energy $\langle y\rangle_{T, \text { test }}$. This value can then be compared to $\langle E\rangle_{T}$ obtained independently by numerical integration of Eq. (24). Tests were carried out by using two constant values of the energy transfer parameter $\alpha(y)$ for $\mathrm{ClOOCl}$ (small molecule, colliding with $\mathrm{N}_{2}$ ) and for 1,1,1-trifluoroethane (intermediate size, colliding with $\mathrm{Kr}$ ). Tests carried out for norbornene (large molecule, colliding with $\mathrm{Kr}$ ) employed an energy-dependent parameter $\alpha(y)$ found from an analysis [36] of shock tube experimental data [44].

The results for $\mathrm{ClOOCl}$ and 1,1,1-trifluoroethane are given in Table I, and those for norbornene are given in Table II. In the tables, $\langle E\rangle_{T}$ is the average thermal energy obtained by numerical integration of Eq. (24), $\alpha$ is the energy transfer parameter, $\langle E\rangle_{\text {new }}$ is the average thermal energy obtained using master equation simulations that incorporate the new normalization method, and $\langle E\rangle_{\text {conv }}$ is the average thermal energy obtained using master equation simulations that incorporate the conventional normalization method. MultiWell utilizes the stochastic simulation algorithm, which has associated errors for a finite number of samples $[45,46]$. The 
Table I Tests of the Simulated Average Thermal Energy

\begin{tabular}{|c|c|c|c|c|}
\hline$T(\mathrm{~K})$ & $\langle E\rangle_{T}\left(\mathrm{~cm}^{-1}\right)$ & $\alpha\left(\mathrm{cm}^{-1}\right)$ & $\langle E\rangle_{\mathrm{conv}}\left(\mathrm{cm}^{-1}\right)$ & $\langle E\rangle_{\text {new }}\left(\mathrm{cm}^{-1}\right)$ \\
\hline \multicolumn{5}{|c|}{$\mathrm{ClOOCl}^{a}$} \\
\hline 200 & 231 & 500 & 259 & 230 \\
\hline 200 & 231 & 100 & 226 & 245 \\
\hline 400 & 825 & 500 & 734 & 831 \\
\hline 400 & 825 & 100 & - & - \\
\hline 800 & 2413 & 500 & 2671 & 2413 \\
\hline 800 & 2413 & 100 & 2261 & 2446 \\
\hline \multicolumn{5}{|c|}{ 1,1,1-Trifluoroethane ${ }^{b}$} \\
\hline 200 & 202 & 500 & 218 & 228 \\
\hline 200 & 202 & 100 & 189 & 240 \\
\hline 400 & 948 & 500 & 874 & 973 \\
\hline 400 & 948 & 100 & 738 & 996 \\
\hline 800 & 3683 & 500 & 3589 & 3691 \\
\hline 800 & 3683 & 100 & 3458 & 3690 \\
\hline 1600 & 11,480 & 500 & 11,474 & 11,507 \\
\hline 1600 & 11,480 & 100 & 11,364 & 11,472 \\
\hline 2400 & 20,482 & 500 & 20,336 & 20,547 \\
\hline 2400 & 20,482 & 100 & 20,302 & 20,458 \\
\hline \multicolumn{5}{|c|}{$1,1,1$-Trifluoroethane ${ }^{c}$} \\
\hline 200 & 202 & 500 & 222 & 222 \\
\hline 200 & 202 & 100 & 180 & 230 \\
\hline 400 & 948 & 500 & 830 & 970 \\
\hline 400 & 948 & 100 & 691 & 991 \\
\hline 800 & 3683 & 500 & 3589 & 3713 \\
\hline 800 & 3683 & 100 & 3410 & 3758 \\
\hline
\end{tabular}

${ }^{a} \Delta E_{\text {grain }}=5 \mathrm{~cm}^{-1}$.

${ }^{b} \Delta E_{\text {grain }}=10 \mathrm{~cm}^{-1}$

${ }^{c} \Delta E_{\text {grain }}=5 \mathrm{~cm}^{-1}$; sampling errors in $\langle E\rangle_{\text {conv }}$ and $\langle E\rangle_{\text {new }}$ are $\leq 1 \%$.

stochastic sampling errors in $\langle E\rangle_{\text {conv }}$ and $\langle E\rangle_{\text {new }}$ presented in Tables I and II are $\leq 1 \%$.

Inspection of Tables I and II shows $\langle E\rangle_{\text {conv }}$ often differs significantly from $\langle E\rangle_{T}$. This is because the three iterations used in the iterative normalization scheme in MultiWell-v.2008.3 are not sufficient for complete numerical convergence. The use of just three iterations is a compromise between achieving reasonable accuracy at higher energies and increasingly inaccurate results at lower energies, as discussed above. The computed

Table II Norbornene ${ }^{a}$

\begin{tabular}{rrcc}
\hline$T(\mathrm{~K})$ & $\langle E\rangle_{T}\left(\mathrm{~cm}^{-1}\right)$ & $\langle E\rangle_{\text {conv }}\left(\mathrm{cm}^{-1}\right)$ & $\langle E\rangle_{\text {new }}\left(\mathrm{cm}^{-1}\right)$ \\
\hline 200 & 207 & 185 & 255 \\
400 & 1476 & 1231 & 1531 \\
800 & 7679 & 7206 & 7642 \\
1200 & 16,602 & 15,555 & 16,569 \\
\hline
\end{tabular}

thermal energy distributions are constrained by both detailed balance and normalization. The computed average thermal energies are different because the conventional method is affected by the problems with normalization and the new method is not. Since the errors in normalization constant $N(y)$ are energy dependent, the energy distributions are in error, resulting in the discrepancies in the average thermal energy $\langle E\rangle_{T}$.

The tables also show that the new approach is more accurate than the conventional one for most combinations of parameters. The following observations come from inspection of the tables:

- $\langle E\rangle_{\text {new }}$ usually tends to be slightly greater than $\langle E\rangle_{T}$.

- Larger values of $\alpha$ tend to give slightly more accurate results for $\langle E\rangle_{\text {new }}$, but not necessarily for $\langle E\rangle_{\text {conv }}$.

- Smaller values of $\Delta E_{\text {grain }}$ tend to improve the accuracy of $\langle E\rangle_{\text {new }}$ at lower temperatures, but that is not always the case at higher temperatures. 
- At higher temperatures, $\langle E\rangle_{\text {new }}$ is uniformly more accurate than $\langle E\rangle_{\text {conv }}$, but that is often not the case at low temperatures.

Since the average thermal energy is a function of temperature, it is interesting to convert the energy errors to temperature differences. For example, at $1200 \mathrm{~K}\langle E\rangle_{\text {conv }}$ for norbornene is in error by -1047 $\mathrm{cm}^{-1}$. This corresponds to a temperature difference $\Delta T=-43 \mathrm{~K}$, or a relative temperature error of $-3.6 \%$. In contrast, the error in $\langle E\rangle_{\text {new }}$ at the same temperature is only $-33 \mathrm{~cm}^{-1}$ and $\Delta T_{\text {new }}=-0.2 \%$. This error is so small that it may just be due to stochastic sampling error, rather than to an intrinsic deficiency in the new approach.

In the second series of tests at two temperatures, 100 samples were taken from each of $10^{4}$ random collision sequences, binned in $10 \mathrm{~cm}^{-1}$ energy grains, normalized, and then displayed for comparison with the exact Boltzmann distribution calculated using Eq. (22). Examples of these tests are shown in Figs. 2 and 3. At both temperatures, the energy distributions computed using the new approach are in excellent agreement with the Boltzmann distribution. Not only are the gross features correct, but most of the detailed structure is reproduced accurately. This performance contrasts with that of the conventional approach (see Fig. 3), which is unable to simulate the rapid oscillations at low energies and misses systematically at higher energies. Note, however, that conventional method simulates the shape of the energy distribution reasonably well at the higher energies.

\section{Energy Transfer Decay}

In this set of tests, simulations were carried out by monitoring the average energy of an ensemble of molecules initially excited to a relatively high energy. Energy transfer was assumed to be governed by an exponentialdown model (Eq. (21)) with energy transfer parameter $\alpha$ that was independent of energy.

The total energy transferred per collision, $\langle\Delta E\rangle_{\text {all }}$ can be written as the sum of the average energies transferred in activating and in deactivating collisions:

$$
\begin{aligned}
\langle\Delta E(y)\rangle_{\text {all }}= & \int_{0}^{y}(x-y) P(x, y) \mathrm{d} x \\
& +\int_{y}^{\infty}(x-y) P(x, y) \mathrm{d} x \\
= & -\langle\Delta E\rangle_{\text {down }}+\langle\Delta E\rangle_{\text {up }}
\end{aligned}
$$

where $\langle\Delta E\rangle_{\text {up }}$ is for activation and $\langle\Delta E\rangle_{\text {down }}$ is for deactivation. For an exponential model with energy transfer parameter $\alpha$ that is independent of energy, it has been shown that $\langle\Delta E\rangle_{\text {all }}$ can be estimated by using

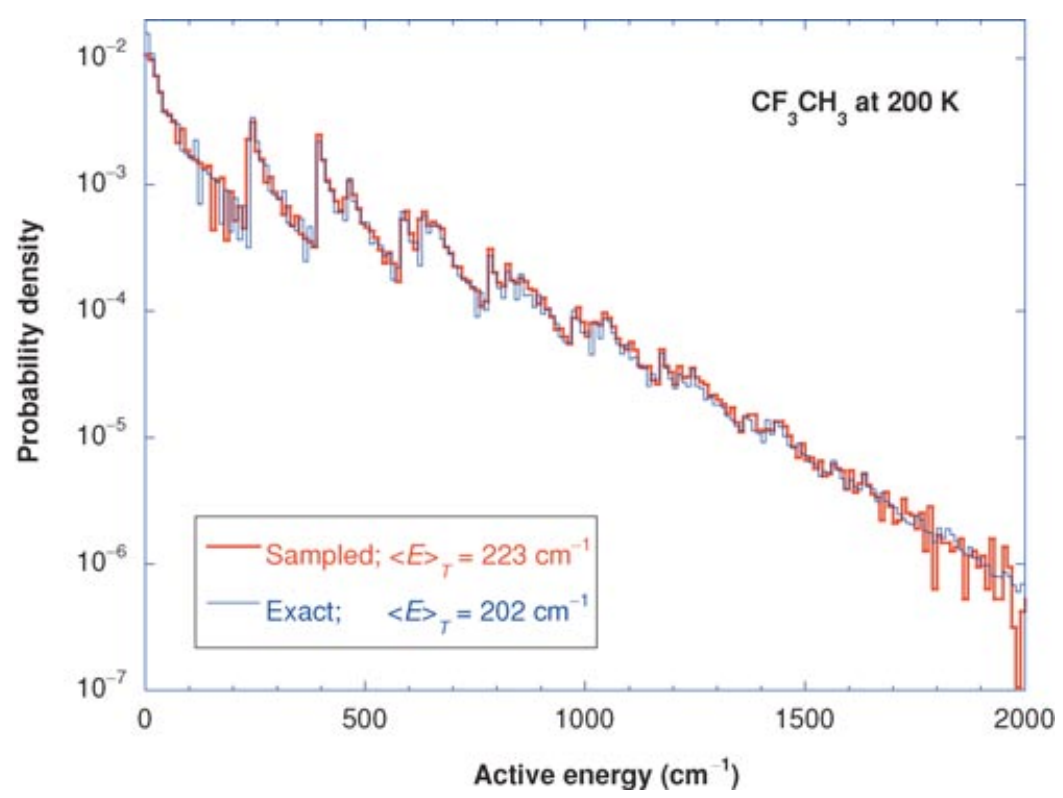

Figure 2 Sampled collision energies from the new method vs. the exact Boltzmann energy distribution at $200 \mathrm{~K}$. Both the thermal energy distribution and the sampled energies were binned in $10 \mathrm{~cm}^{-1}$ energy grains. Statistical fluctuations in the sampled energies are apparent at the highest energies shown. The average thermal energies differ by only $\sim 21 \mathrm{~cm}^{-1}$. Most of the detailed energy structure in the exact distribution is reproduced in the sampled distribution. [Color figure can be viewed in the online issue, which is available at www.interscience.wiley.com.] 


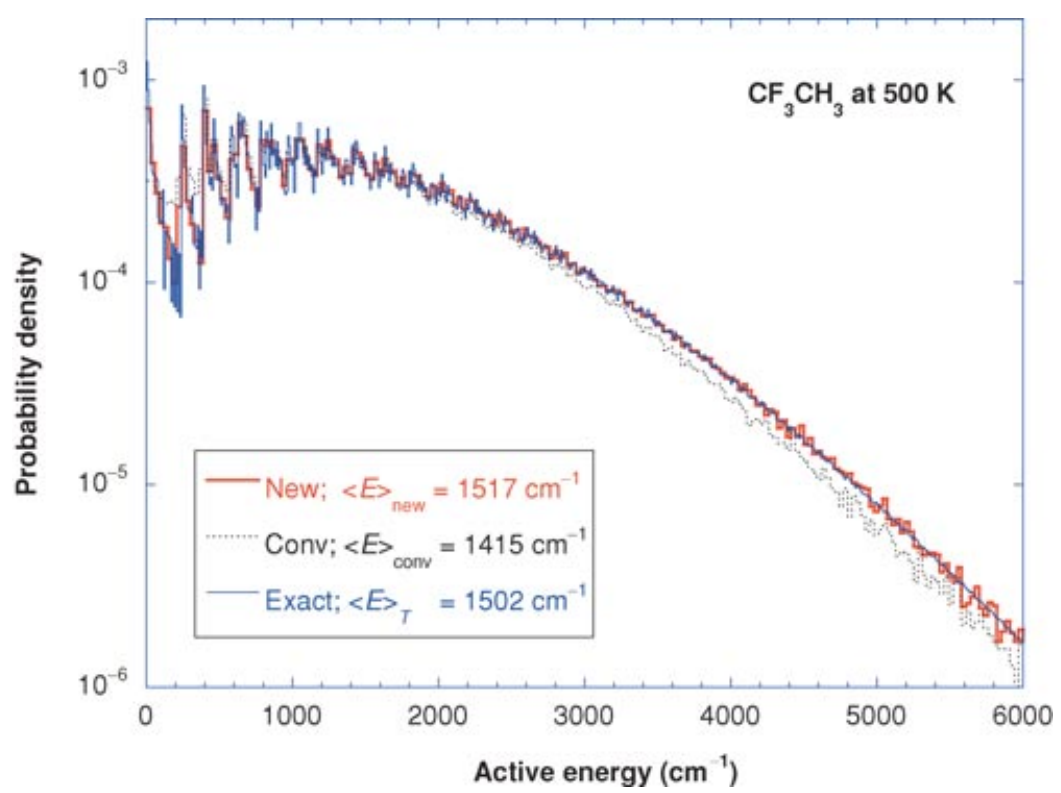

Figure 3 Sampled collision energies vs. exact thermal energy distribution at $500 \mathrm{~K}: 10^{6}$ energy samples (100 samples in each of $10^{4}$ stochastic trials). The thermal energy distribution is shown with $10 \mathrm{~cm}^{-1}$ energy grains, and the sampled energies were binned in $30 \mathrm{~cm}^{-1}$ energy grains. The average thermal energy obtained using the new method $\langle E\rangle_{\text {new }}$ is only $\sim 15 \mathrm{~cm}^{-1}$ different from the exact value. The old, conventional method gives $\langle E\rangle_{\text {conv }}$ in error by $\sim 100 \mathrm{~cm}^{-1}$. Most of the detailed energy structure in the exact distribution is reproduced using the new method, but not by the conventional method. [Color figure can be viewed in the online issue, which is available at www.interscience.wiley.com.]

the following equation [47]:

$$
\left\langle{ }^{2} E(y)\right\rangle_{\mathrm{all}} \approx\left[1 / k_{B} T+1 / \alpha-B\right]^{-1}-\alpha
$$

where $B=\mathrm{d}(\ln (\rho)) / \mathrm{d} y$. From Eq. (25), it is apparent that $\langle\Delta E\rangle_{\text {all }}$ is nearly constant as long as $y>\alpha$.

When $\langle\Delta E\rangle_{\text {all }}$ is nearly constant, the resulting decay of average energy is expected to be a nearly linear function of time. This expectation is confirmed by the simulations shown in Fig. 4. The initial energy decay rate obtained using the conventional approach is $3.0 \times 10^{8}$ $\mathrm{cm}^{-1} \mathrm{~s}^{-1}$, and at the simulated pressure of 0.1 Torr the collision frequency is $2.9 \times 10^{6} \mathrm{~s}^{-1}$. Together, these values give $\langle\Delta E\rangle_{\text {all }}=103 \mathrm{~cm}^{-1}$, using the conventional approach. The estimate from Eq. (25c) is in good agreement with this value: $\langle\Delta E\rangle_{\mathrm{all}, 24 c}=109 \mathrm{~cm}^{-1}$. This estimate is based on $\alpha=200 \mathrm{~cm}^{-1}, T=$ $300 \mathrm{~K}$, and $B=6.41 \times 10^{-4}\left(\mathrm{~cm}^{-1}\right)^{-1}$, which was found from a least-squares fit of the toluene density of states from 35,000 to $40,000 \mathrm{~cm}^{-1}$.

The initial energy decay rate obtained using the new approach is $4.6 \%$ slower than the simulation that used the conventional approach. This minor difference is mostly due to the difference in collision frequencies. As discussed above, the new approach adopts $k_{\mathrm{LJ}}$ at a reference energy and assumes the collision rate constant $k_{c}$ is proportional to the normalization constant. At the internal energy of $40,000 \mathrm{~cm}^{-1}$, the normal- ization constant is $5.6 \%$ smaller than at the reference energy ( $4990 \mathrm{~cm}^{-1}$ in these simulations), resulting in a slower collision frequency and hence a slower energy decay rate. After correcting for the change in collision frequency, the simulation with the new approach gives $\langle\Delta E\rangle_{\text {all }}=105 \mathrm{~cm}^{-1}$, in very good agreement with the conventional method, as expected for relatively high energies when $\alpha$ is a constant.

Figure 4 shows that simulations carried out with constant $\alpha$ are in very good agreement over the entire energy decay. The simulations carried out using the energy-dependent $\alpha$ (Fig. 5) also show very good agreement. Here the simulations are more realistic, since the energy transfer parameter $\alpha(y)$ is the energy-dependent function determined in kinetically controlled selective ionization (KCSI) experiments [48]. Most of the difference between the two approaches arises from minor differences in collision frequency. It is clear that experimental data can be fitted equally well using either method, and the fitted parameters will differ because of the energy-dependent inelastic collision rate coefficient.

\section{Unimolecular Reactions in Shock Waves}

In shock wave experiments, the reactant gas mixture consists of a buffer gas and a few percent or less of a reactant gas. The gas mixture is subjected to a shock wave, which adiabatically compresses the gas mixture 


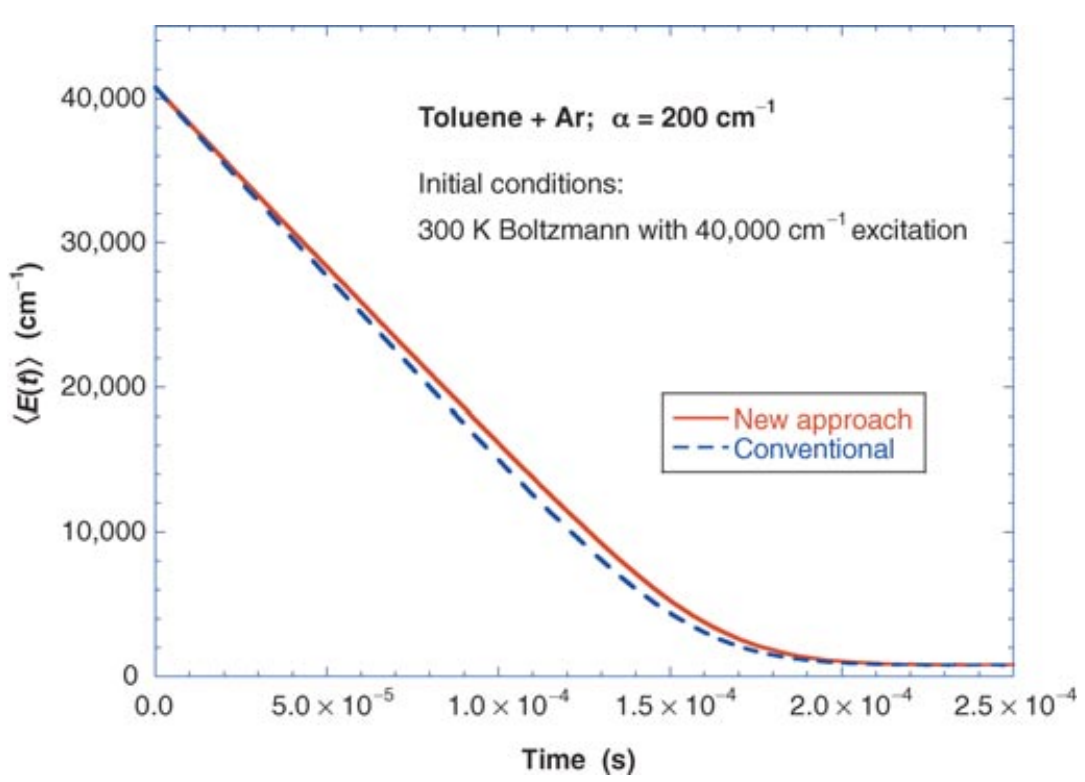

Figure 4 Simulated deactivation of excited toluene in 0.1 Torr of argon at $300 \mathrm{~K}$, assuming the energy transfer parameter $\alpha=200 \mathrm{~cm}^{-1}$ (independent of energy). [Color figure can be viewed in the online issue, which is available at www.interscience.wiley.com.]

and raises its translational temperature on a submicrosecond time scale [49]. Thus the reactant, initially at thermal equilibrium with the bath at ambient temperature, finds itself immersed in a bath at much higher temperature. The reactant, initially with a vibrational energy distribution that corresponds to the initial ambient temperature, undergoes collisions until it either decomposes or its energy distribution reaches equilibrium with the new translational temperature.
In the shock tube experiments of Kiefer et at. [44], the reactant was norbornene and the bath gas was krypton. The experiments were later modeled successfully by Barker and King [36], who used a master equation that was similar in many respects to MultiWell.

To accurately model shock tube experiments is a very demanding task for master equation simulations. Not only must the chemical reaction be modeled accurately, but the whole evolution of the energy

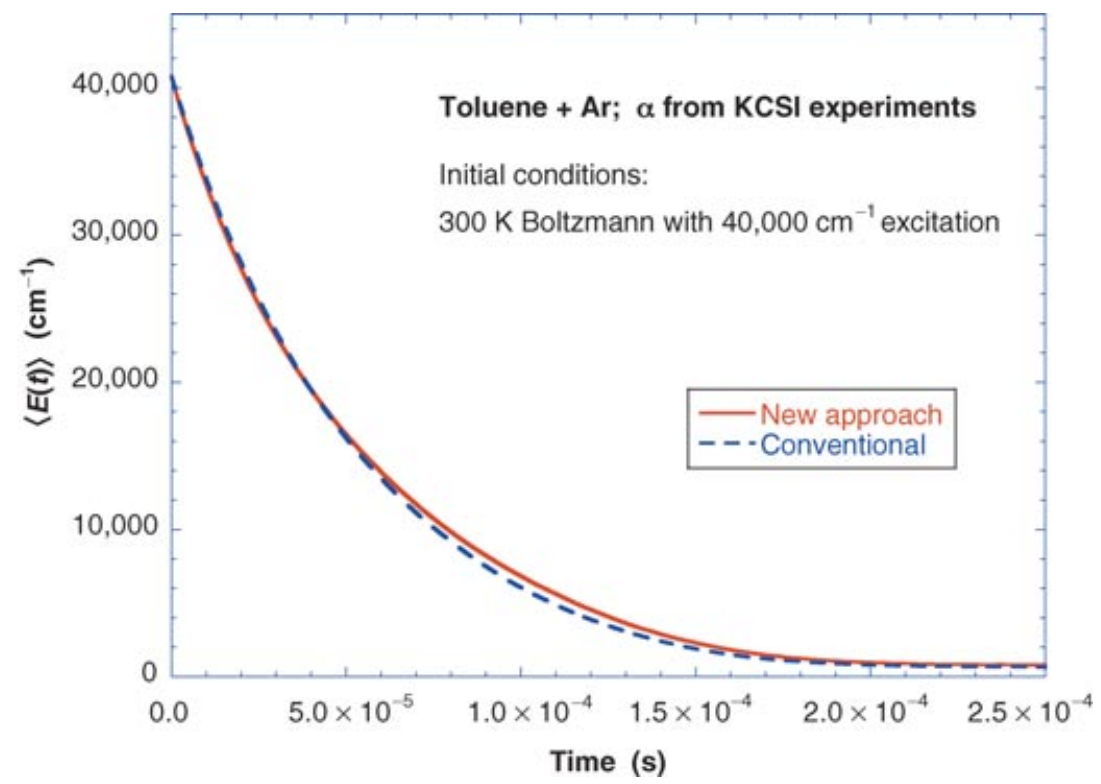

Figure 5 Simulated deactivation of excited toluene in 0.1 Torr of argon at $300 \mathrm{~K}$, assuming the energy transfer parameter $\alpha(y)$ obtained in KCSI experiments [48]. [Color figure can be viewed in the online issue, which is available at www.interscience.wiley.com.] 
distribution must be modeled accurately as it evolves from the initial low-temperature thermal distribution to the final steady-state ("fall-off") distribution that is the result of competition between collisional energy transfer and chemical reaction. Thus the master equation model must be accurate essentially all of the way from near the reactant zero-point energy to high above the reaction threshold energy.

Experimental knowledge about the evolving energy distribution comes from several kinds of information $[36,44]$. These include the pressure- and temperaturedependent unimolecular reaction rate constants $\left(k_{\text {uni }}\right)$ measured after the energy distribution has established the final steady state. But more direct dynamical information on energy transfer comes from the measurement of the rate of collisional energy transfer that accompanies the evolution of the energy distribution and the closely related incubation time $\left(\tau_{\text {inc }}\right)$. The reaction incubation time is defined as the time delay from the arrival of the incident shock wave to the onset of reaction. This time delay arises because of the time needed to collisionally activate the molecule to energies above the reaction threshold.

In the tests performed here, the model parameters found in our previous work [36] are used with the conventional and new approaches to ascertain the impact of the new approach. As discussed above, the two approaches are expected to give somewhat different results. Since master equation models are only approximations to the true physico-chemical system, we are not in a position to determine which model is more correct. Thus the only question to be addressed here is, can the new approach be used successfully to describe the experimental data?

To answer that question, results obtained using the conventional and new approaches are first compared, and then new calculations are carried out using the new approach but with revised parameters to determine whether the new approach can accurately model the results obtained using the conventional one with the original parameters. The energy transfer model chosen for present purposes is Model \#3 from Barker and King [36]: the exponential-down model with $\alpha(y)=40+0.0063 y$. The specific experiment that is used for illustration is Shock \#76 reported by Kiefer et al. [44] and previously modeled in Barker and King [36].

The calculated fraction of norbornene that survives is plotted as a function of time in Fig. 6 for both the conventional and new approaches. Qualitatively, the two approaches are similar to the previous model calculations carried out by Barker and King [36], but clearly the two approaches differ quantitatively as expected for an energy-dependent $\alpha(y)$. To extract quantitative

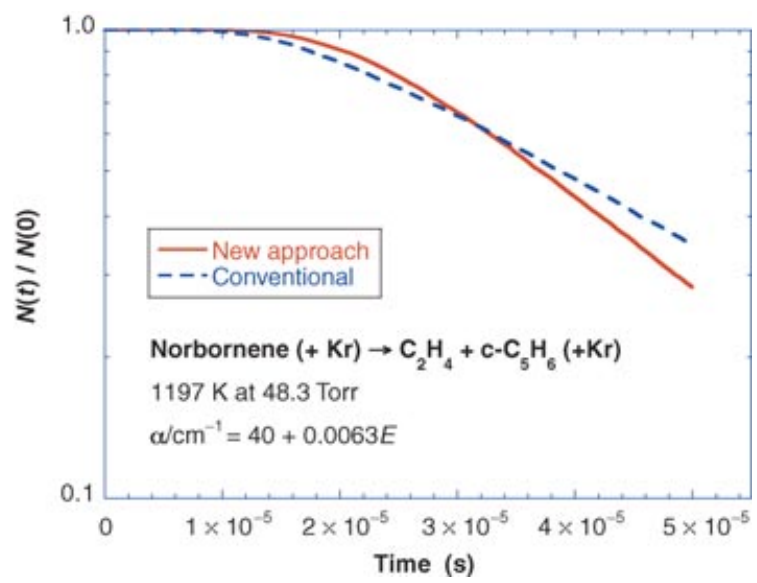

Figure 6 Simulation of norbornene decomposition in shock waves. The conditions correspond to Shock \#76 reported by Kiefer et al. [44] and modeled previously by Barker and King [36]. [Color figure can be viewed in the online issue, which is available at www.interscience.wiley.com.]

estimates of the steady-state unimolecular rate constant $k_{\text {uni }}$ and incubation time $\tau_{\text {inc }}$, it is convenient to fit the data in Fig. 6 by using the following function:

$\frac{N(t)}{N(0)}=\exp \left\{\left[-k_{\text {uni }}\left(t-\tau_{\text {inc }}\right)\right][1-\exp (-c t)]^{b}\right\}$

where parameters $c$ and $b$ (which typically take values of $\sim 1000$ and 0.2 , respectively) empirically describe the time evolution of $k_{\text {uni }}$ from zero to its final steadystate value. This is the correct form of Eq. (9) in Barker and King [36] (which contained typographical errors).

From least-squares fits of the data in Fig. 6, the steady-state values for $k_{\text {uni }}$ are $2.69 \times 10^{4} \mathrm{~s}^{-1}$ and $3.56 \times 10^{4} \mathrm{~s}^{-1}$ (at $1197 \mathrm{~K}$ and 48.3 Torr of $\mathrm{Kr}$ bath gas) for the conventional and the new approach, respectively; the corresponding values for $\tau_{\text {inc }}$ are 7.8 and $10.2 \mu \mathrm{s}$, respectively. The differences between these two results are due to several factors. First, the new approach gives a better description of the thermal energy distribution, resulting in differences in the computed steady-state unimolecular rate constants. Second, because the energy transfer parameter $\alpha(y)$ is a function of energy, the normalization constant is a function of energy, even in the upper energy regime. Therefore, the constant, energy-independent collision frequency in the conventional approach is replaced by an energydependent function, resulting in differences in the vibrational relaxation time and incubation time.

The key question is whether or not the new approach can be empirically parameterized to fit the experimental data. Since the data were originally modeled by using the conventional approach, we assume the conventional model is a reasonable description of the 
experimental data. By varying parameters, we found that by using $\alpha(y)=70+0.003 y$, the new approach reproduces the results obtained with the conventional method, with deviations smaller than the width of the heavy dashed line in Fig. 6. This result shows that the new approach is just as effective at fitting experimental data as the conventional one, even for shock tube experiments, which are highly sensitive to the accurate treatment of collisional energy transfer. The numerical values of the parameters are different, but this is mostly because the inelastic collision frequency is no longer assumed to be independent of energy.

\section{CONCLUSIONS}

The ultimate goal of this work is to improve the accuracy of master equation simulations. The specific aims of this paper were to introduce a plausible new physical assumption about the inelastic collision frequency, to determine whether the new approach is a significant improvement over the old, and to ascertain whether the new approach will still allow one to fit experimental data empirically. A new assumption is needed because the old assumption that the rate constant for inelastic collisions $k_{c}(y)$ is independent of energy, $y$, results in normalization constants, $N(y)$, that are nonphysical at low energies, or ill-behaved numerically.

The plausible new assumption is that the rate constant for inelastic collisions $k_{c}(y)$ is directly proportional to the normalization factor for the collision stepsize distribution $N(y)$. This assumption is qualitatively correct, since both $k_{c}(y)$ and $N(y)$ depend on energy, $y$. However, little is known about $k_{c}(y)$. For that reason, we arbitrarily postulate a direct proportionality, but this point will have to be addressed again in the future. To use parameters that have magnitudes that are similar to previous conventional master equation simulations, we define a reference energy, $E_{\text {ref }}$, at which $k_{c}\left(E_{\text {ref }}\right)$ is assumed to equal the rate constant for Lennard-Jones collisions, $k_{\mathrm{LJ}}$.

Simulations based on the new approach show that it eliminates the nonphysical and ill-behaved normalization constants, which motivated this work. They also show that the new approach does a significantly better job over-all in simulating the Boltzmann distribution function. Simulations with empirically adjusted parameters were capable of fitting the previous conventional simulations of several experiments, showing that the new approach is capable of fitting experimental data.

On the basis of these considerations, we have adopted this new approach in our own master equation simulations. We expect this new approach will also be useful in 2D ( $E, J$ resolved) master equation implementations, where the numerical ill-behavior may be even worse than in the 1D master equation studied here. The new approach performs better than the conventional model, but it must eventually be replaced when knowledge of the total inelastic collision frequency is improved. That, however, may not happen for some time to come.

Thanks go to Jingyao Liu (Jilin University) for providing calculated anharmonicity coefficients and the hindered internal rotation barrier for $\mathrm{ClOOCl}$, to Yi Jie Chua, an undergraduate researcher here at Michigan, for assistance with some preliminary calculations, and to Andrea Maranzana (University of Torino) for useful discussions.

\section{APPENDIX A: CRITERIA IN MULTIWELL FOR $\mathrm{E}_{\text {ref }}$}

For a description of double arrays and for definitions of terms, see the MultiWell User Manual [25].

In MultiWell, we identify $E_{\text {ref }}$ with the critical energy of the lowest reaction threshold energy (when multiple reaction channels are involved) that is higher in energy than the energy boundary (parameter Emax 1) between the lower and upper portions of the double array. If no reaction threshold energies are below Emax1, we arbitrarily specify $E_{\text {ref }}$ as equal to Emax 1 . At Emax $1, N(y)$ is a relatively smooth function and the density of states is typically $>10-100$ states $/ \mathrm{cm}^{-1}$.

\section{APPENDIX B: PARAMETERS FOR THE MASTER EQUATION SIMULATIONS}

Each excited species was treated approximately as a symmetrical top with a 1D K-rotor and a 2D adiabatic rotation. All densities of states were calculated using the "active energy," which consists of the K-rotor and the vibrations; because of angular momentum conservation in the unimolecular reactions, the $2 \mathrm{D}$ rotation is omitted from the density of states calculation in the most common treatments of angular momentum $[1,3]$. Since the same density of states is used in the detailed balance expression for inelastic collisions, the energy transfer parameters refer to the active energy and not to pure vibrational or rotational transitions.

Vibrational frequencies for $\mathrm{ClOOCl}$ were taken from Jacobs et al. [50] with vibrational anharmonicities calculated using density functional theory (B3LYP/6$311+\mathrm{G}(3 \mathrm{df})$ ) [Jingyao Liu, private communication, 2008]. The lowest vibrational frequency $\left(127 \mathrm{~cm}^{-1}\right)$ 
Table AI ClOOCl Vibrational Frequencies

\begin{tabular}{lc}
\hline$\omega^{\circ}\left(\mathrm{cm}^{-1}\right)$ & $\omega_{e} X_{e}$ \\
\hline 754 & -9.329 \\
648. & -3.061 \\
543 & 3.889 \\
419. & 0.069 \\
321. & -1.695 \\
127. & Hindered rotor barrier $=5493 \mathrm{~cm}^{-1 a}$ \\
& Moment of inertia \\
$46.32 \mathrm{amu} \AA^{2}$ & (K-rotor) \\
\hline
\end{tabular}

${ }^{a}$ Twofold internal rotation.

Table AII Lennard-Jones Parameters

\begin{tabular}{llcc}
\hline Species & $\sigma(\AA)$ & $\varepsilon / k_{B}(\mathrm{~K})$ & Reference \\
\hline $\mathrm{N}_{2}$ & 3.74 & 82 & {$[51]$} \\
$\mathrm{Kr}$ & 3.61 & 190 & {$[52]$} \\
$\mathrm{ClOOCl}$ & 4.84 & 367 & Estimated \\
$1,1,1-\mathrm{CF}_{3} \mathrm{CH}_{3}$ & 4.959 & 387 & {$[52]$} \\
Norbornene & 5.5 & 330 & {$[44]$} \\
\hline
\end{tabular}

was treated as a twofold hindered internal rotation with hindrance barrier height $5493 \mathrm{~cm}^{-1}$ [Jingyao Liu, private communication, 2008]. These parameters are summarized in Table AI.

Vibrational frequencies, moments of inertia, and other parameters can be found in previous papers on 1,1,1-trifluoroethane [34] and norbornene [36]. Lennard-Jones parameters for all of the collision partners are summarized in Table AII.

\section{BIBLIOGRAPHY}

1. Forst, W. Unimolecular Reactions. A Concise Introduction; Cambridge University Press: Cambridge, UK, 2003.

2. Gilbert, R. G.; Smith, S. C. Theory of Unimolecular and Recombination Reactions; Blackwell Scientific: Oxford, UK, 1990.

3. Holbrook, K. A.; Pilling, M. J.; Robertson, S. H. Unimolecular Reactions; Wiley: Chichester, UK, 1996.

4. Barker, J. R.; Golden, D. M. Chem Rev 2003, 103, 45774591.

5. Barker, J. R.; Yoder, L. M.; King, K. D. J Phys Chem A 2001, 105, 796-809.

6. Hocker, L. O.; Kovacs, M. A.; Rhodes, C. K.; Flynn, G. W.; Javan, A. Phys Rev Lett 1966, 17, 233-235.

7. Weitz, E.; Flynn, G. W.; Ronn, A. M. J Chem Phys 1972, 56, 6060-6067.

8. Smith, G. P.; Barker, J. R. Chem Phys Lett 1981, 78, 253-258.

9. Hippler, H.; Troe, J.; Wendelken, H. J Chem Phys Lett 1981, 84, 257-259.
10. Jalenak, W.; Weston, R. E., Jr.; Sears, T. J.; Flynn, G. W. J Chem Phys 1985, 83, 6049-6050.

11. Lohmannsroben, H. G.; Luther, K. Chem Phys Lett 1988, 144, 473.

12. Liu, C.-L.; Hsu, H.-C.; Lyu, J.-J.; Ni, C.-K. J Chem Phys 2006, 124, 54302.

13. Flynn, G. W.; Parmenter, C. S.; Wodtke, A. M. J Phys Chem 1996, 100, 12817.

14. Krajnovich, D. J.; Parmenter, C. S.; Catlett, D. L., Jr. Chem Rev 1987, 87, 237-288.

15. Hold, U.; Lenzer, T.; Luther, K.; Reihs, K.; Symonds, A. C. J Chem Phys 2000, 112, 4076-4089.

16. Lenzer, T.; Luther, K.; Reihs, K.; Symonds, A. C. J Chem Phys 2000, 112, 4090-4110.

17. Hold, U.; Lenzer, T.; Luther, K.; Symonds, A. C. J Chem Phys 2003, 119, 11192-11211.

18. Durant, J. L.; Kaufman, F. Chem Phys Lett 1987, 142 , 246-251.

19. Xue, B.; Han, J.; Dai, H.-L. Phys Rev Lett 2000, 84, 2606-2609.

20. Yardley, J. T. Introduction to Molecular Energy Transfer; Academic Press: New York, 1980.

21. Herzfeld, K. F.; Litovitz, T. A. Absorption and Dispersion of Ultrasonic Waves; Academic Press: New York, 1959.

22. Schwartz, R. N.; Slawsky, Z. I.; Herzfeld, K. F. J Chem Phys 1952, 20, 1591.

23. Havey, D. K.; Liu, Q.; Li, Z.; Elioff, M.; Fang, M.; Neudel, J.; Mullin, A. S. J Phys Chem A 2007, 111, 2458-2460.

24. Barker, J. R. Int J Chem Kinet 2001, 33, 232-245.

25. Barker, J. R.; Ortiz, N. F.; Preses, J. M.; Lohr, L. L.; Maranzana, A.; Stimac, P. J.; Nguyen, L. T. Ann Arbor, MI. Available at http://aoss.engin.umich.edu/multiwell/, 2009. The current and all prior versions of the software are available from the Web site.

26. Weston, R. E., Jr.; Barker, J. R. J Phys Chem A. 2006, 110, 7888-7897.

27. Forst, W. Theory of Unimolecular Reactions; Academic Press: New York, 1973.

28. Barker, J. R. Chem Phys 1983, 77, 301-318.

29. Gilbert, R. G.; King, K. D. Chem Phys 1980, 49, $367-$ 375.

30. Troe, J. J Chem Phys 1977, 66, 4745-4757.

31. Hippler, H.; Schranz, H. W.; Troe, J. J Phys Chem 1986, 90, 6158-6167.

32. Lenzer, T.; Luther, K.; Troe, J.; Gilbert, R. G.; Lim, K. F. J Chem Phys 1995, 103, 626-641.

33. Troe, J. Chem Rev 2003, 103, 4565-4576.

34. Barker, J. R.; Stimac, P. J.; King, K. D.; Leitner, D. M. J Phys Chem A 2006, 110, 2944-2954.

35. Shi, J.; Barker, J. R. Int J Chem Kinet 1990, 22, 187-206.

36. Barker, J. R.; King, K. D. J Chem Phys 1995, 103, 4953 4966.

37. Whitten, G. Z.; Rabinovitch, B. S. J Chem Phys 1963, 38, 2466

38. Barker, J. R. In Highly Excited States: Relaxation, Reaction, and Structure; Mullin, A.; Schatz, G. C. (Eds.); 
American Chemical Society: Washington DC, 1997; pp. 220-236.

39. Barker, J. R. Ber Bunsen-Ges Phys Chem 1997, 101, 566.

40. Barker, J. R.; Ortiz, N. F.; Preses, J. M.; Lohr, L. L.; Maranzana, A.; Stimac, P. J.: Ann Arbor, MI. Available at http://aoss.engin.umich.edu/multiwell/, 2008.

41. Beyer, T.; Swinehart, D. F. Comm Assoc Comput Machines 1973, 16, 379.

42. Stein, S. E.; Rabinovitch, B. S. J Chem Phys 1973, 58, 2438-2445.

43. Lenzer, T.; Luther, K. Phys Chem Chem Phys 2004, 6, 955-959.

44. Kiefer, J. H.; Kumaran, S. S.; Sundaram, S. J Chem Phys 1993, 99, 3531-3541.
45. Gillespie, D. T. J Comp Phys 1976, 22, 403-434.

46. Gillespie, D. T. J Phys Chem 1977, 81, 2340-2361.

47. Barker, J. R.; Golden, R. E. J Phys Chem 1984, 88, 1012.

48. Grigoleit, U.; Lenzer, T.; Luther, K.; Mutzel, M.; Takahara, A. Phys Chem Chem Phys 2001, 3, 21912202.

49. Kiefer, J. H.; Buzyna, L. L.; Dib, A.; Sundaram, S. J Chem Phys 2000, 113, 48-58.

50. Jacobs, J.; Kronberg, M.; Muller, H. S. P.; Willner, H. J Am Chem Soc 1994, 116, 1106-1114.

51. Hippler, H.; Troe, J.; Wendelken, H. J. J Chem Phys 1983, 78, 6709-6717.

52. Kiefer, J. H.; Katapodis, C.; Santhanam, S.; Srinivasan, N. K.; Tranter, R. S. J Phys Chem A 2004, 108, 24432450 . 